\title{
Assessment of potential strain injury to rectus capitis posterior minor muscles during whiplash type distortions of the cervical spine
}

https://doi.org/10.1515/jom-2021-0094

Received March 25, 2021; accepted May 10, 2021;

published online June 4, 2021

\begin{abstract}
Context: Whiplash type injuries resulting from a rear end motor vehicle accident (REMVA) are thought to be caused by excessive loading and displacement of structural components of the cervical spine. On impact, the seat propels the driver's torso forward relative to the head, resulting in forced flexion of the occipitoatlantal (OA) joint, accompanied by forced stretching of the rectus capitis posterior minor (RCPm) muscles. Flexion of the OA joint and stretching of the RCPm muscles continues to increase until the vehicle's headrest strikes the back of the driver's head. It is known that externally applied forces that attempt to move the OA joint beyond its anatomic barrier can result in fracture, dislocation, or soft tissue damage to its structural components. However, the magnitude of headrest backset, defined as the distance between the driver's head and the vehicle's headrest, that would result in RCPm muscles being stretched to a length that would put them at risk for a muscle strain injury is unknown.
\end{abstract}

Objectives: To quantify the relationships among flexion of the OA joint, RCPm muscle stretch, and backset, and to quantify the biomechanical response of RCPm muscles to increasing levels of axial load due to stretching.

Methods: Unembalmed head and neck specimens from three White females aged 85, 63, and 70 years were obtained from the Anatomical Services Division at the University of Maryland. Donors had provided written consent allowing use of their body for research purposes. Using an analytic model of the OA joint, the relationships between

\footnotetext{
*Corresponding author: Richard Hallgren, PhD, Department of Physical Medicine \& Rehabilitation, Michigan State University, B411 West Fee Hall, 909 Wilson Road, East Lansing, MI 48824-2604, USA, E-mail: hallgren@msu.edu Jacob J. Rowan, DO, Department of Osteopathic Manipulative Medicine, Michigan State University, East Lansing, MI, USA
}

flexion of the OA joint and RCPm muscle stretch as a function of backset were estimated. RCPm muscles were removed from the cadavers and forcibly stretched using a servomechanism controlled hydraulic testing machine to quantify the load/displacement properties. After testing, the tissues were sectioned, mounted, and stained using Masson's trichrome to selectively stain muscle fibers red and collagen blue.

Results: Forced flexion of the OA joint was seen to be directly related to the magnitude of headrest backset. For values of backset greater than $7.2 \mathrm{~cm}$, biomechanical testing of the RCPm muscles revealed that strain injuries ranged from the tearing of a few muscle fibers to complete rupture of the muscle and separation of the tendon at the posterior process of $\mathrm{C} 1$.

Conclusions: Results showed that headrest backset at the time of vehicle impact is an important factor in estimating the risk of muscle strain injury to RCPm muscles. Muscle strain injury would be expected to impact the functional relationship between the RCPm muscles and the pain sensitive spinal dura. Physicians should be alert to the possibility that cervicogenic pain patients who have experienced whiplash associated with REMVA may show clinically relevant structural damage to the RCPm muscles on MRI.

Keywords: anatomic barrier; car accident; cervical spine; muscle strain; rectus capitis posterior minor; whiplash.

When a car is struck from behind in a rear end motor vehicle accident (REMVA), the car's seat back propels the driver's torso forward [1]. During the first 50-100 ms after impact, which is defined as the retraction phase, the inertia of the driver's head causes it to remain fixed in the sagittal plane without rotation while the driver's torso moves forward relative to the head (Figure 1) [2, 3]. As the relative distance between the torso and the head increases, forced flexion of the occipitoatlantal (OA) joint and forced stretching of the rectus capitis posterior minor (RCPm) muscles occurs [4, 5]. Forced flexion of the OA joint and stretching of the RCPm muscles ceases at the end of the 


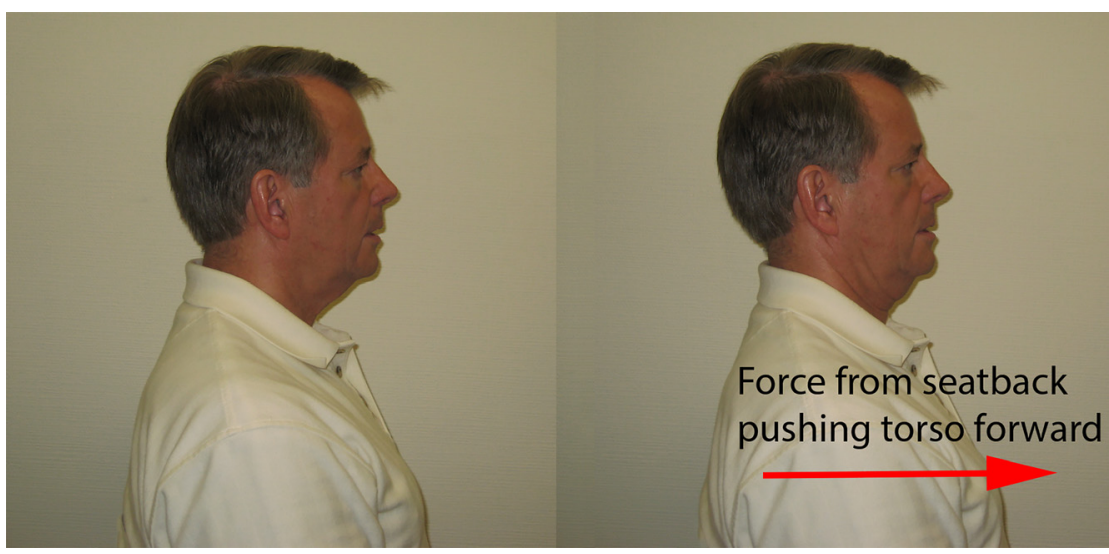

Figure 1: Simulated forward motion of the torso of a 74 year old man relative to his head, resulting in flexion of the occipitoatlantal (OA) joint and stretching of the rectus capitis posterior minor muscles. retraction phase of the REMVA, when the driver's head strikes the headrest.

Flexion of the OA joint during the retraction phase of a REMVA is very similar to flexion of the OA joint during voluntary retraction of the head. Both result in a characteristic S-shaped curve resulting from flexion at the OA joint and extension at the lower levels of the cervical spine [5]. One significant difference is that forces producing flexion of the OA joint during a REMVA are applied over a shorter time period than those that produce flexion of the OA joint during voluntary head retraction.

Risk of a muscle strain injury to the RCPm muscles is directly related to the magnitude of $\mathrm{OA}$ joint flexion. Angular displacement of the OA joint is directly related to the backset, which is defined as the distance between the driver's head and the headrest at the moment of vehicle impact (Figure 2) [6, 7]. Whiplash type injuries caused by unrestrained, rapid backward and forward movement of the head are thought to be a source of neck strain/sprain injuries [8]. Injury to structures innervated by the C1 to C3 spinal nerves - which include upper cervical synovial joints, muscles, C2-3 discs, and the dura mater - can result in referred pain to the head, commonly diagnosed as cervicogenic headache [9]. The authors of a previous simulation study [10] hypothesized that minimizing the backset

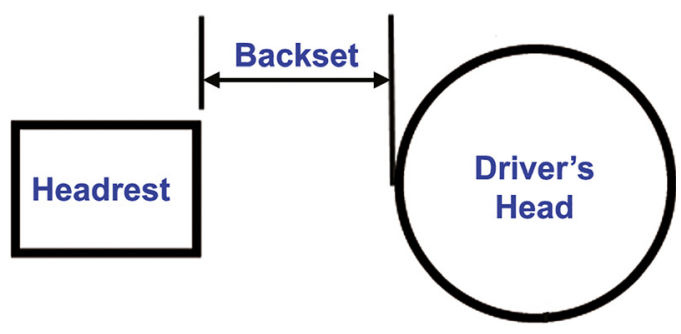

Figure 2: Illustration of backset, which is the distance between the driver's head and the headrest at the moment of vehicle impact. should reduce the number of whiplash type injuries sustained in REMVA.

Physiologic joint motion is defined by a range of active and passive movement. The total range of motion (ROM) of a joint is defined by its anatomic barrier. If external forces such as those experienced during a REMVA overcome the resistive forces of muscle, tendons, and ligaments, forcing the joint past the anatomic barrier, fracture, dislocation, and tearing of soft tissues such as muscles, tendons, and ligaments can or will occur. "Muscle strain injury" is a term that physicians use to describe injury to a muscle that typically results from forced stretching. The injury is typically graded as minor (Grade II) or major (Grade III) [11]. Minor strain injuries usually repair themselves in a few days [12], while major strain injuries result in tearing of the muscle and do not spontaneously repair themselves. Trauma of the latter magnitude, which is seen in rotator cuff tears [13], significantly impacts the functional capacity of the injured muscle. The term "strain" is used by biomedical engineers to quantify the percentage change in length of a material in relation to its original length as it is stretched [14]. The term "muscle strain" then refers to a normalized metric quantified by subtracting the original length of a muscle from its final length and dividing the result by the original length [14]. For the purposes of this study, the original length of RCPm muscles signifies their length when the driver has their head positioned in a self-selected, neutral head posture (NHP). The final length is dependent on the OA joint angle at the instant the headrest strikes the driver's head, which is dependent on the backset (Figure 2) when REMVA occurs. The "muscle strain" quantity is then expressed as a percentage and allows for comparison of the biomechanical response of muscles while accounting for normal morphological variations among subjects; muscle strain injuries begin to occur when that value exceeds a certain percentage [1]. 
RCPm muscles arise from a narrow tendon that attaches to the posterior tubercle on the posterior arch of $\mathrm{C} 1$ and inserts into the occipital bone. Electromyographic (EMG) activation patterns of these muscles suggest that they stabilize the OA joint by helping maintain congruency of the joint surfaces [15]. Because of their small size, their functional significance might be discounted if it were not for a myodural bridge that interconnects them to the pain sensitive spinal dura $[16,17]$. The orientation of connective tissue fibers and the linear displacement of the dura mater with increasing muscle tension suggest that the RCPm muscles modulate force distribution within the dura mater during flexion, extension [18], and retraction of the head $[19,20]$. A prior study [21] showed that mechanical stretching of the dura resulted in referred headache. The neuroanatomic basis for referred headache depends on convergence of cervical and trigeminal afferents on common neurons in the trigeminocervical nucleus [9]. Any structure, such as the spinal dura, that is innervated by the upper three cervical nerves can therefore become a source of referred pain. The role of the myodural bridge in the initiation and maintenance of headache is uncertain, but structural damage to the RCPm muscles, such as might occur during a REMVA, would have an adverse effect on their functional role in preventing excessive dural tension.

The objectives of this study were to quantify the relationships among flexion of the OA joint, RCPm muscle stretch, and backset, and to quantify the biomechanical response of RCPm muscles to increasing levels of axial load due to stretching.

\section{Methods}

\section{Study period}

This project began in 2010 with the acquisition, dissection, and biomechanical testing of RCPm muscles. Immediately following testing, the muscle tissues were fixed, sectioned, and mounted onto glass slides for analysis. In 2020, with the advent of the coronavirus 2019 pandemic, we found that our clinical research slowed, which provided time to revisit the archived digital data and the slides, at which point we performed the analysis reported here.

\section{Estimation of RCPm muscle length as a function of $O A$ joint angle}

A biomechanical model of the human skull (C0), the atlas (C1), and the RCPm muscles [22] was used to calculate the amount of flexion of the $\mathrm{OA}$ joint that would occur at the end of the retraction phase in a REMVA as a function of backset. This was used to estimate both the length of the RCPm muscles as a function of joint angle and the magnitude of RCPm muscle strain that would occur. When the backset is equal to $0 \mathrm{~cm}$, the headrest is assumed to be touching the driver's head and the driver is assumed to have an NHP, which corresponds to an $\mathrm{OA}$ angle of $14^{\circ}$ [23]. Flexion of the $\mathrm{OA}$ joint increases the joint angle and results in stretching of the RCPm muscles. The following equation provides an estimate of RCPm muscle length $(L)$ as a function of the OA joint angle $(\alpha)$.

$L=2^{\star} R^{\star} \sin (\alpha / 2)=$ estimated change in length $(\mathrm{mm})$ of RCPm muscles as a function of change in OA joint angle (degrees)

In the equation, $R$ is the distance measured from the origin of the axis of rotation of $\mathrm{C} 1$ about $\mathrm{C} 0$ to the attachment point on $\mathrm{C} 1$ of the RCPm muscle (Figure 3). $R$ is estimated to be equal to $28 \mathrm{~mm}$ with an NHP [24]. $\alpha$ is the $=$ change in OA joint angle, defined as the difference between the joint angle at the time of impact and the joint angle at the time when the subject's head strikes the head restraint.

The magnitude of RCPm muscle strain at the end of the retraction phase of a REMVA as a function of backset is shown in Figure 4.

\section{Biomechanical testing}

Unembalmed head and neck specimens from 3 White females aged 85, 63, and 70 years were obtained from the Anatomical Services Division at the University of Maryland. Donors had provided written consent allowing use of their body for research purposes. The Neurosurgical Atlas [25] provides a detailed, stepwise dissection of the suboccipital region, and dissection for this study was performed according to it. First, a wedge of occipital bone with the tendinous attachment of the left RCPm muscle and a separate wedge of bone containing the tendinous attachment of the right RCPm muscle were removed. A section of the posterior arch of $\mathrm{C} 1$ containing the inferior attachment of both the left and right RCPm muscles was then removed (Figure 5).

A manual, side action, tensile grip was fabricated by Michigan State University to securely hold the bony fragments while testing [26] so that the muscles would be stretched in pure tension by a servomechanism controlled hydraulic testing machine (model 1331; Instron, Norwood, MA) at strain rates of 0.1 and 1,000 times the resting

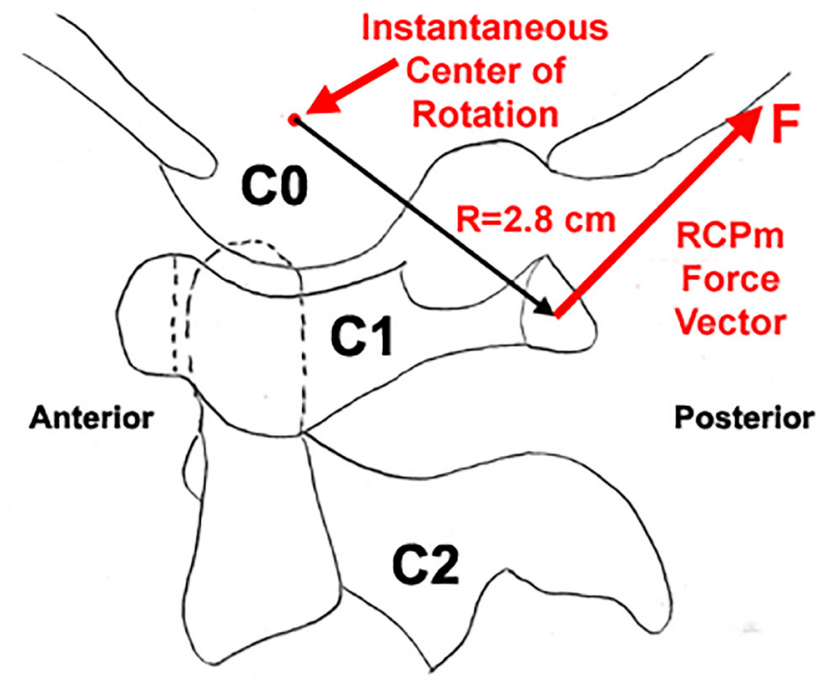

Figure 3: Instantaneous axis of rotation of the posterior process of $\mathrm{C} 1$ about $\mathrm{C} 0$, showing length of moment arm (R). 


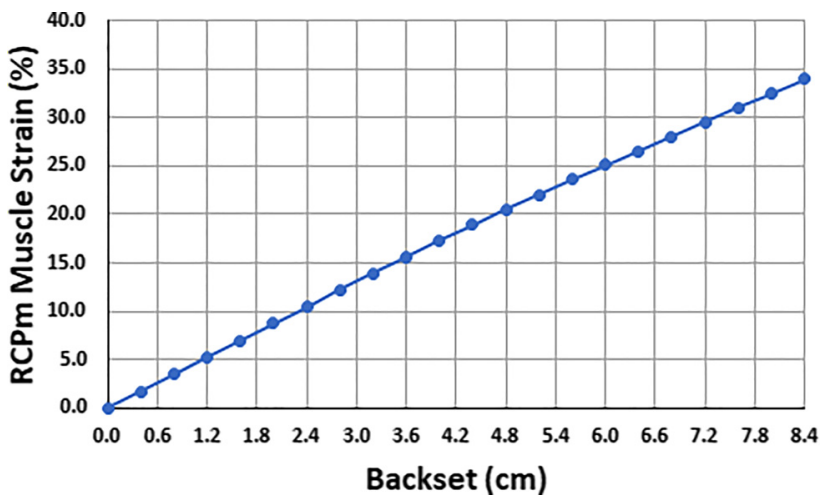

Figure 4: Rectus capitis posterior minor muscle strain at the end of the retraction phase of a rear end motor vehicle accident as a function of backset (the distance between the driver's head and the headrest at the moment of impact). When the backset is equal to $0 \mathrm{~cm}$, the headrest is touching the driver's head and the driver is assumed to have a neutral head posture, where the occipitoatlantal joint angle is equal to $14^{\circ}$.

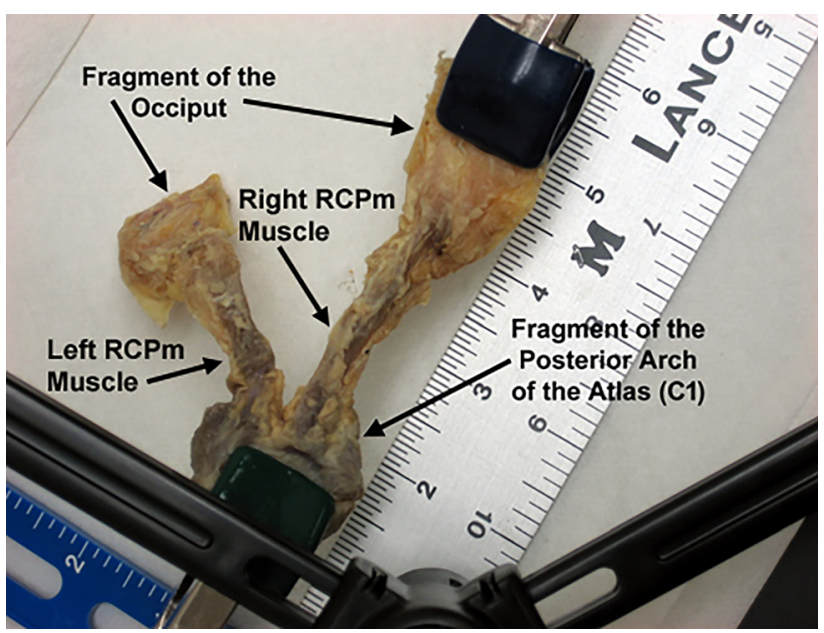

Figure 5: Specimen from a 63 year old White female cadaver, showing the right rectus capitis posterior minor muscle stretched to a length consistent with the maximum occipitoatlantal joint angle at the anatomic barrier to motion.

length per second. Each of our three samples was subjected to increasing levels of strain $(20,30,40,50 \%)$ to determine when the specimen transitioned from a region of elastic deformation to a region of plastic deformation (Figure 6). This level of strain was used to define the magnitude of muscle strain that would result in irreversible injury to the muscle.

\section{Histology}

Immediately following the final biomechanical tests, the muscle tissues were processed and embedded into super molds. Blocks were trimmed and $4 \mu$ to $5 \mu$ sections were then taken every $250 \mu \mathrm{m}$ and placed on glass slides. Sections were stained using Masson's
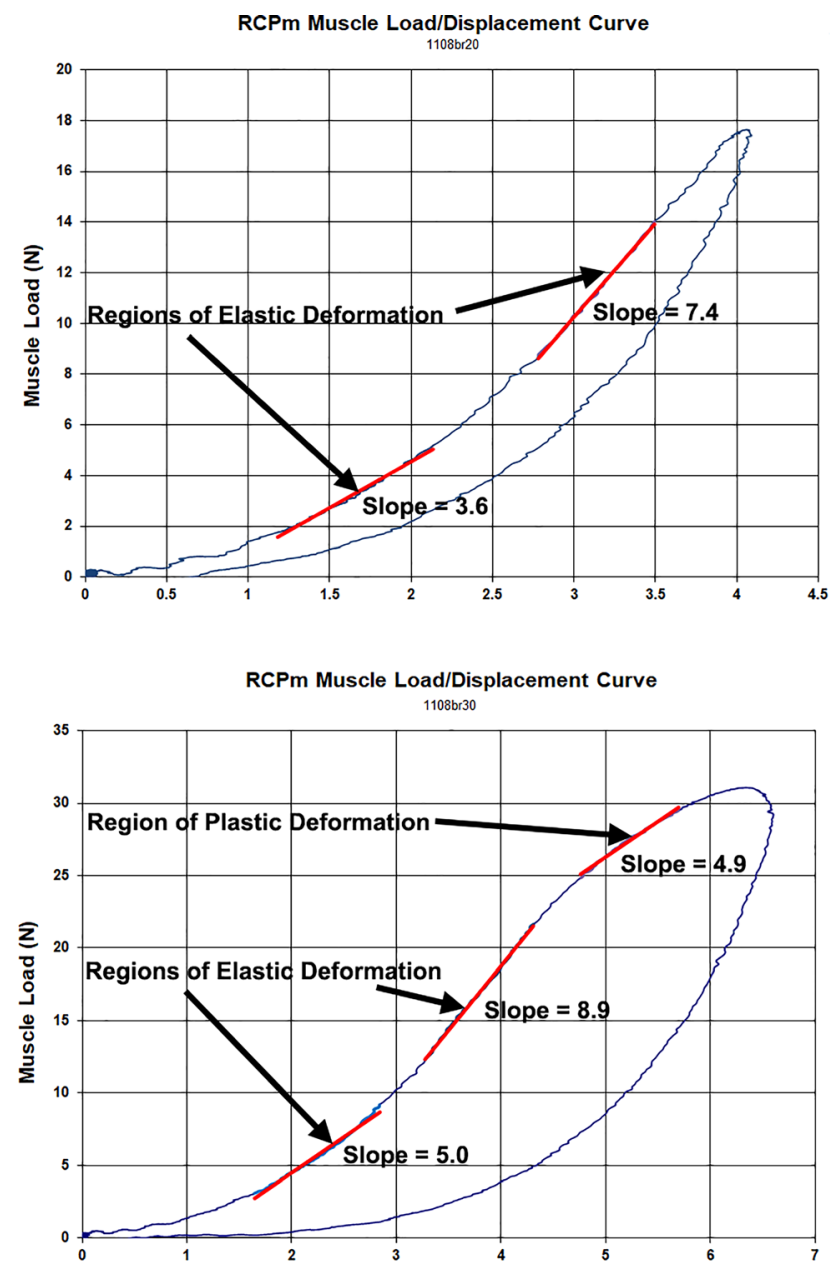

Figure 6: (A, top) Load/displacement plot of rectus capitis posterior muscle from subject 1108 in this study, showing typical viscoelastic characteristics at $20 \%$ muscle strain. Notice that the slope of the curve is always increasing, indicating that elastic deformation is occurring. (B, bottom) Load/displacement plot showing biomechanical characteristics at $30 \%$ muscle strain. Notice that the slope in the elastic region of the curve becomes steeper with increasing values of muscle displacement up to $4 \mathrm{~mm}$. However, at $5 \mathrm{~mm}$, the slope begins to decrease, indicating that plastic deformation is occurring. Plastic deformation is characterized by tearing of the muscle tissues.

trichrome staining protocol to help distinguish muscle fibers (red) from collagen (blue) [27]. Tissues were then digitized using a digital microscope (Keyence Corp) at X30 and the images were analyzed using ImageJ software [28].

\section{Results}

Forced flexion of the OA joint was seen to be directly related to the magnitude of headrest backset. For values of backset greater than $7.2 \mathrm{~cm}$, biomechanical testing of the RCPm 


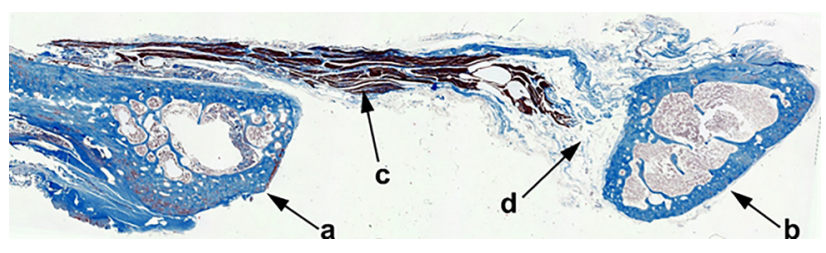

Figure 7: The rectus capitis posterior muscle from subject 1108 in this study is shown, stretched to failure. The fragment of the occiput is on the left (a) and the fragment of the posterior arch of $\mathrm{C} 1$ is on the right (b). The tissues stained red are muscles; the tissues stained blue are collagen. Tearing of the muscle fascicles can be seen at the center (c). Tearing of the tendon away from the posterior process can be seen on the right $(d)$.

muscles revealed that strain injuries ranged from the tearing of a few muscle fibers to complete rupture of the muscle and separation of the tendon at the posterior process of $\mathrm{C} 1$. Levels of muscle strain and load were found to be consistent with values predicted by finite element (FE) models at simulated REMVA impact greater than $5 \mathrm{~g}$ [29].

\section{Discussion}

For levels of muscle strain less than $20 \%$, published laboratory tests [30, 31] have shown that cyclic stretching does not result in changes in biomechanical parameters of rabbit skeletal muscle, which is consistent with the results of our study (Figure 6A), in which RCPm muscles exhibited a load/displacement response that is typical of viscoelastic tissues. At levels of muscle strain greater than $30 \%$ (Figure 6B), the load/displacement curve of the muscles in our study was seen to transition from a region of elastic deformation to a region of irreversible plastic deformation resulting from tearing of muscle fascicles, as indicated by the decrease in the slope of the curve at muscle lengths greater than $5 \mathrm{~mm}$. The implication is that, for our samples, the injury threshold occurred at a muscle strain of $30 \%$, with increasing levels of tissue damage occurring at increasing levels of muscle strain up to 50\% (Figure 7).

Increasing levels of tissue damage are significant when considering the functional tissue link that interconnects RCPm muscles to the pain sensitive spinal dura [16, 17]. While the role of the myodural bridge as a source of chronic headache pain is uncertain, it is certain that structural damage to the RCPm muscles would have an adverse effect on their functional relationship.

RCPm muscle strain is directly related to flexion of the $\mathrm{OA}$ joint, which is directly related to headrest backset. Risk of a whiplash type injury is therefore directly related to the backset and to the intensity of the impact $[6,7]$. Values of backset, measured when the driver is engaged in normal driving activities, have been shown to be significantly larger than values of backset measured when the vehicle is stationary [32]. This is a result of drivers protruding their heads and assuming a forward head posture (FHP) while driving. Shugg et al. [33] reported that 12 of 14 subjects maintained an approximate backset of $7.8 \mathrm{~cm}$ (standard deviation, 24.8) while driving, a value that exceeds the recommended guideline of $5.5 \mathrm{~cm}$ set by the National Highway Traffic Safety Administration [34]. Our analysis (Figure 4) predicted a value of muscle strain equal to $24 \%$ for a backset of $5.5 \mathrm{~cm}$. If a driver were to protrude their head so that the backset was equal to $7.8 \mathrm{~cm}$, our model predicted that they would exceed the $30 \%$ injury threshold for skeletal muscle reported in prior studies that established it at 31\% [35], 30\% [36], and 38\% [37]. These values are consistent with values reported by Hedenstierna et al. [29] but are more conservative than values reported by Stemper et al. [38]. While the exact magnitude of threshold is open to discussion, our model shows that reducing backset, either by adjusting the headrest or reducing FHP while driving, should reduce the risk of a strain injury to the RCPm muscles from a REMVA.

The results of our study revealed that high levels of muscle strain, consistent with whiplash distortions, cause tearing of RCPm muscle fascicles and separation of the tendon at the posterior process of $\mathrm{C}$. Muscle strain rates between 10 and $100 \mathrm{~cm} / \mathrm{s}$ have been reported to produce similar results in the tibialis anterior muscles of rabbits [39]. Normally, injured muscles repair themselves in a few days. In the instance of a rotator cuff tear resulting in FI of the muscles characterized on magnetic resonance imaging (MRI) by edema and inflammation [40], spontaneous repair of the muscle does not always occur. The longer the condition persists, the more severe the FI becomes, accompanied by greater loss of function [13]. In those cases, surgery is used to repair the tear, halting FI and restoring function [41]. Hallgren and Rowan [42] demonstrated that the image resolution on MRI is sufficient to evaluate fine structures within the $\mathrm{OA}$ interspace, potentially enabling diagnosis of muscle strain injury to RCPm muscles [42]. Unfortunately, the standard clinical protocol for the cervical spine does not include the OA interspace, and the relatively high cost of MRI has restricted its use as a diagnostic tool. As a result, muscle strain injury to RCPm muscles may be underdiagnosed.

Evidence of muscle tearing should lead the clinician to explore a specific exercise or treatment plan. Anecdotal evidence suggests that head retraction exercises have a beneficial effect for patients with head and neck pain [43]. Concentric, eccentric, and isometric contractions have 
been shown to be effective in strengthening muscle [44]. If it is assumed that pathology in RCPm muscles is not due to muscle disease or neurogenic atrophy, then selectively exercising RCPm muscles would be expected to result in an increase in cross section area (CSA) and increased strength. Increased RCPm muscle strength would be expected to restore normal musculoskeletal functionality of this critical component of the upper cervical spine, with a commensurate decrease in headache pain and FHP. We hypothesize that the combined use of osteopathic manipulative treatment and focused head exercises (FHE) would increase the CSA of RCPm muscles on MRI and decrease headache measures in female patients diagnosed with cervicogenic headache.

\section{Limitations}

Due to a lack of young cadaveric material, our sample was limited to three elderly female specimens. We stretched the muscle samples starting from their resting length, assuming that the driver would have their head positioned in a self selected NHP. However, the specific biomechanical properties of $\mathrm{RCPm}$ muscles are variable and the compliance of these muscles would most likely vary at shorter lengths. Wide variations in morphologic and kinematic properties of the upper cervical spine, along with variations of architectural properties among muscles, make it challenging to construct an analytical model that would accurately define absolute levels of muscle strain that would result in a muscle strain injury at the musculotendinous junction of RCPm muscles among the general population [45]. Since our samples were not living, their biomechanical response reflects only the passive response to an external force. Living tissue has the option to actively respond to a force that attempts to stretch it, absorbing greater amounts of energy and reducing its susceptibility to injury [46]. Therefore, we might expect to see increased incidence of injury occurring at values of backset less than what the study predicts.

\section{Conclusions}

The results of our study demonstrate that reducing the distance between the driver's head and the headrest at the time of vehicle impact is an important factor in protecting RCPm muscles from muscle strain injury. For values of backset greater than $7.2 \mathrm{~cm}$, whiplash distortions have the potential to result in muscle strain injuries that would range from the tearing of a few muscle fibers to complete rupture of the muscle and separation of the tendon at the posterior process of $\mathrm{C} 1$. A muscle strain injury would be expected to significantly impact the functional relationship between the RCPm muscles and the pain sensitive spinal dura. Physicians should be alert to the possibility that cervicogenic pain patients who have experienced whiplash type injuries associated with a REMVA may show clinically relevant structural damage to the RCPm muscles on MRI that may account for the difficulty in managing their pain.

Research funding: None reported.

Author contributions: Both authors provided substantial contributions to conception and design, acquisition of data, or analysis and interpretation of data; Dr. Hallgren drafted the article or revised it critically for important intellectual content; both authors gave final approval of the version of the article to be published; and both authors agree to be accountable for all aspects of the work in ensuring that questions related to the accuracy or integrity of any part of the work are appropriately investigated and resolved.

Competing interests: None reported.

\section{References}

1. Vasavada, AN, Brault, JR, Siegmund, GP. Musculotendon and fascicle strains in anterior and posterior neck muscles during whiplash injury. Spine 2007;32:756-65.

2. O'Neill, B. Head restraints - the neglected countermeasure. Accid Anal Prev 2000;32:143-50.

3. Viano, DC. Seat properties affecting neck responses in rear crashes: a reason why whiplash has increased. Traffic Inj Prev 2003;4:228-39.

4. Grauer, JN, Panjabi, MM, Cholewicki, J, Nibu, K, Dvorak, J. Whiplash produces an s-shaped curvature of the neck with hyperextension at lower levels. Spine 1997;22:2489-94.

5. Cholewicki, J, Panjabi, MM, Nibu, K, Babat, LB, Grauer, JR, Dvorak, J. Head kinematics during in vitro whiplash simulation. Accid Anal Prev 1998;30:469-79.

6. Krafft, M, Kullgren, A, Ydenius, A, Tingvall, C. Influence of crash pulse characteristics on whiplash associated disorders in rear impacts - crash recording in real life crashes. Traffic Inj Prev 2002;3:141-9.

7. Cormier, J, Gwin, L, Reinhart, L, Wood, R, Bain, C. A comprehensive review of low-speed rear impact volunteer studies and a comparison to real-world outcomes. Spine 2018; 43:1250-8.

8. Quinlan, KP, Annest, JL, Myers, B, Ryan, G, Hill H. Neck strains and sprains among motor vehicle occupants - United States, 2000. Accid Anal Prev 2004; 36:21-7.

9. Bogduk, N. Cervicogenic headaches: anatomic basis and pathophysiologic mechanisms. Curr Pain Headache Rep 2001;5: 382-6.

10. Ivancic, PC, Sha, D, Panjabi, MM. Whiplash injury prevention with active head restraint. Clin Biomech 2009;24:699-707. 
11. Speer, KP, Lohnes, J, Garrett, WE. Radiographic imaging of muscle strain injury. Am J Sports Med 1993;21:89-95.

12. Yang, KH, King, Al. Neck kinematics in rear-end impacts. Pain Res Manage 2003;8:79-85.

13. Melis, B, Nemoz, C, Walch, G. Muscle fatty infiltration in rotator cuff tears: descriptive analysis of 1688 cases. Orthop TraumatolSur 2009;95:319-24.

14. Garrett, WE, Safran, MR, Seaber, AV, Glisson, RR, Ribbeck, BM. Biomechanical comparison of stimulated and nonstimulated skeletal muscle pulled to failure. Am J Sports Med 1987;15: 448-54.

15. Hallgren, RC, Rowan, JJ. Implied evidence of the functional role of the rectus capitis posterior muscles. J Am Osteopath Assoc 2020; 120:395-403.

16. Hack, GD, Koritzer, RT, Robinson, WL, Hallgren, RC, Greenman, $\mathrm{PE}$. Anatomical relationship between the rectus capitis posterior minor muscle and the dura mater. Spine 1995;20:2484-6.

17. Sillevis, R, Hogg, R. Anatomy and clinical relevance of sub occipital soft tissue connections with the dura mater in the upper cervical spine. Peerl 2020;8:e9716.

18. Venne, G, Rasquinha, BJ, Kunz, M, Ellis, RE. Rectus capitis posterior minor: histological and biomechanical links to the spinal dura. Spine 2017;42:E466-73.

19. Hallgren, RC, Rowan, JJ. Forward head posture and activation of rectus capitis posterior muscles. J Am Osteopath Assoc 2017;117: 24-31.

20. Jiang, W-B, Zhang, Z-H, Yu, S-B, Sun, J-X, Ding, S-W, Ma, G-J, et al. Scanning electron microscopic observation of myodural bridge in the human suboccipital region. Spine 2020;45:E1296-301.

21. Fontaine, D, Almairac, F, Santucci, S, Fernandez, C, Dallel, R, Pallud, J, et al. Dural and pial pain-sensitive structures in humans: new inputs from awake craniotomies. Brain 2018;41: 1040-48.

22. Hallgren, RC. Modeling length-tension properties of RCPm muscles during voluntary retraction of the head. Man Ther 2014; 19:319-23.

23. Ordway, NR, Seymour, RJ, Donelson, RG, Hojnowski, LS, Edwards, T. Cervical flexion, extension, protrusion, and retraction: a radiographic segmental analysis. Spine 1999;24:240-7.

24. Dugailly, PM, Sobczak, S, Moiseev, F, Sholukha, V, Salvia, P, Feipel, V, et al. Musculoskeletal modeling of the suboccipital spine: kinematics analysis, muscle lengths, and muscle moment arms during axial rotation and flexion extension. Spine 2011;36: E413-22.

25. Cohen-Gadol, A. Suboccipital muscles: stepwise dissection. The Neurosurgical Atlas, 2020. https://www.neurosurgicalatlas. com/neuroanatomy/suboccipital-muscles-stepwise-dissection.

26. Hallgren, RC. Injury threshold of rectus capitis muscles at the atlanto-occipital joint. J Manip Physiol Ther 2017;40:71-6.

27. Dey, P. Connective tissue stain: principle and procedure. In: Basic and Advanced Laboratory Techniques in Histopathology and Cytology, Singapore: Springer. 2018. https://doi.org/10.1007/ 978-981-10-8252-8_10.

28. Schneider, CA, Rasband, WS, Eliceiri, KW. NIH Image to Image): 25 years of image analysis, Nat Methods 2012;9:671-5, PMID 22930834.
29. Hedenstierna, S, Halldin, P, Siegmund, GP. Neck muscle load distribution in lateral, frontal, and rear-end impacts. Spine 2009; 34:2626-33.

30. Tsuang, YH, Sun, JS, Chen, IH, Hsu, SH, Tsao, KY, Wei, KY, et al. The effects of cyclic stretching on tensile properties of the rabbit's skeletal muscle. Clin Biomech 1998;13:48-53.

31. Rehorn, MR, Schroer, AK, Blemker, SS. The passive properties of muscle fibers are velocity dependent. J Biomech 2014;47: 687-93.

32. Jonsson, B, Stenlund, H, Bjornstig, U. Backset - stationary and during car driving. Traffic Inj Prev 2008;9:568-73.

33. Shugg, JAJ, Vernest, K, Dickey, JP. Head restraint backset during routine automobile driving: drivers usually exceed the recommended guidelines. Traffic Inj Prev 2011;12:180-6.

34. National Highway Traffic Safety Administration. Federal motor vehicle safety standards; Head restraints; Final Rule, (2010) 49 CFR Part 571, Federal Register Vol. 75, page 67246.

35. Sun, JS, Hang, YS, Tsuang, YH, Cheng, CK, Tsao, KY, Hsu, SW. Morphological changes of the triceps surae muscle tendon unit during passive extension: an in vivo rabbit model. Clin Biomech 1998;13:634-40.

36. Brooks, SV, Faulkner, JA. Severity of contraction-induced injury is affected by velocity only during stretches of large strain. J Appl Physiol 2001;91:661-6.

37. Hang, YS, Tsuang, YH, Sun, JS, Cheng, CK. Failure of stimulated skeletal muscle mainly contributed by passive force: an in vivo rabbit model. Clin Biomech 1996;11:343-7.

38. Stemper, BD, Yoganandan, N, Pintar, FA. Effect of head restraint backset on head-neck kinematics whiplash. Accid Anal Prev 2006;38:317-23.

39. Nikolaou, PK, MacDonald, BL, Glisson, RR, Seaber, AV, Garrett, WE. Biomechanical and histological evaluation of muscle after controlled strain injury. Am J Sports Med 1987;15:9-14.

40. Gerber, C, Meyer, DC, Frey, E, von Rechenberg, B, Hoppeler, H, Frigg, $R$, et al. Reversion of structural muscle changes caused by chronic rotator cuff tears using continuous musculotendonous traction. An experimental study in sheep. J Shoulder Elbow Surg 2007;18:163-71.

41. Melis, B, Wall, B, Walch, G. Natural history of infraspinatus fatty infiltration in rotator cuff tears. J Shoulder Elbow Surg 2010;19: 757-63.

42. Hallgren, RC, Rowan, JJ. Magnetic resonance imaging parameters selected for optimal visualization of the occipitoatlantal interspace. J Am Osteopath Assoc 2019;119: 173-82.

43. Lee, MY, Jeon, H, Choi, JS, Park, Y, Ryu,JS. Efficacy of modified cervical and shoulder retraction exercise in patients with loss of cervical lordosis and neck pain. Ann Rehabil Med 2020;44:210-7.

44. Roig, M, O’Brien, K, Kirk, G, Murray, R, McKinnon, P, Shadgan, B, et al. The effects of eccentric verses concentric resistance training on muscle strength and mass in healthy adults: a systematic review with meta-analysis. Br J Sports Med 2009;43:556-68.

45. Garner, BA, Pandy, MG. Estimation of musculotendon properties in the human upper limb. Ann Biomed Eng 2003;31:207-20.

46. Garrett, WE. Muscle strain injuries. Am J Sports Med 1996;24: S2-8. 